

\title{
Non-conservative forces in bulk systems
}

Todorov, T. N., Cunningham, B., Dundas, D., \& Horsfield, A. P. (2017). Non-conservative forces in bulk systems. Materials Science And Technology. https://doi.org/10.1080/02670836.2017.1296991

\author{
Published in: \\ Materials Science And Technology
}

Document Version:

Peer reviewed version

Queen's University Belfast - Research Portal:

Link to publication record in Queen's University Belfast Research Portal

Publisher rights

(C) 2017 Informa UK Limited trading as Taylor \& Francis Ltd.

This work is made available online in accordance with the publisher's policies. Please refer to any applicable terms of use of the publisher.

\section{General rights}

Copyright for the publications made accessible via the Queen's University Belfast Research Portal is retained by the author(s) and / or other copyright owners and it is a condition of accessing these publications that users recognise and abide by the legal requirements associated with these rights.

Take down policy

The Research Portal is Queen's institutional repository that provides access to Queen's research output. Every effort has been made to ensure that content in the Research Portal does not infringe any person's rights, or applicable UK laws. If you discover content in the Research Portal that you believe breaches copyright or violates any law, please contact openaccess@qub.ac.uk. 


\title{
Non-conservative forces in bulk systems
}

\author{
Tchavdar N. Todorov, Brian Cunningham, and Daniel Dundas \\ Atomistic Simulation Centre, School of Mathematics and Physics, \\ Queen's University Belfast, Belfast BT7 1NN, United Kingdom \\ Andrew P. Horsfield \\ Department of Materials and Thomas Young Centre, \\ Imperial College London, London $S W^{r}$ 2AZ, United Kingdom
}

(Dated: November 16, 2016)

\begin{abstract}
The ability of electrons and phonons to equilibrate is fundamental to the thermodynamic and transport properties of solids. Nonetheless in mesoscopic systems sufficiently high current densities can lead to situations where the phonon subsystem can no longer reach a steady state. In previous work this phenomenon was connected with the demonstration that interatomic forces under current are non-conservative, with dramatic dynamical effects. Here we consider these effects in bulk systems. We arrive at a critical current density beyond which current flow results in the uncompensated stimulated emission of a blast of phonons. The result is illustrated with simulations of long atomic wires. It is hoped that the paper will stimulate research into the possible relevance of the effect to electropulsing.

PACS numbers: 72.10.Bg, 73.23.-b, 73.63.Nm
\end{abstract}

Keywords: General formulation of transport theory; Electronic transport in mesoscopic systems; Quantum wires; Current-induced forces; Electromigration 


\section{INTRODUCTION}

We can see and feel - and even hear and smell - the effects of current flow in a lightbulb. Atomic and molecular wires - the systems of interest in the field of Molecular Electronics [1] - can carry current densities 6-7 orders of magnitude larger than those in a lightbulb filament. As a result, both electromigration forces [2] and local heating [3] in these systems can be dramatic.

We have certain intuitive expectations when considering current-driven atomic motion. One of them was highlighted in a visionary article by Sorbello [4]: the effective force field experienced by scattering centres in an electron flow should be non-conservative even under ideal steady-state conditions. If the current density is spatially non-uniform, then we can design closed paths such that the electron wind force does non-zero net work on scatterers when they travel around these closed paths. This notion later led to the direct construction - through non-equlibrium openboundary molecular dynamics - of the single-atom analogue of a waterwheel, where current in an atomic wire with a bend drives the corner atom around, pumping energy into the atom during its directional circular motion [5].

A second analogy comes from a natural phenomenon. Suppose we see a picture of a lake with waves running across it. We would know immediately that the picture was taken on a windy day, with the direction of the wind furthermore being evident from the direction of the waves. This is also a form of energy transfer with a directional sense: the flow generates a forward jet of propagating waves.

These ideas have been explored recently in long atomic wires, demonstrating that the action of non-conservative interatomic forces under current can be connected with the preferential directional emission of travelling phonons [6, 7], as illustrated schematically in Fig. 1.

The aim of the present study is to apply these ideas to bulk conductors. The next section shows that there is a critical current density, above which it becomes possible for an electron current to generate explosive directional phonon jets, through uncompensated stimulated emission. Section III illustrates the resultant dynamics by model simulations of long one-dimensional conductors. Concluding comments are given in section IV.

\section{WATERWHEEL EFFECT IN BULK CONDUCTORS}

Our starting point is the framework set out in Ref. [8]. We consider two degenerate harmonic phonon modes, with generalised displacement operators $\hat{X}, \hat{Y}$ and annihilation operators $\hat{A}_{X}, \hat{A}_{Y}$, 


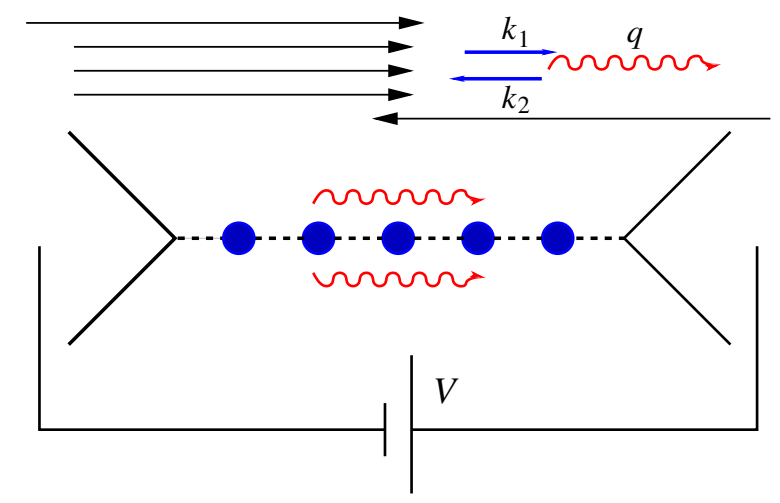

FIG. 1: Schematic of the generation of a forward-travelling phonon shower in a current-carrying atomic wire. The incoming electron current, created by an applied electrochemical potential difference, emits phonons by inelastic electron backscattering. The imbalance in occupancy between electronic states with opposite velocities results in the directional bias of the emitted phonons.

in a steady-state current-carrying system. Ref. [8] envisages localised vibrations in a mesoscopic conductor but the analysis is generic. Next we form linear combinations of the two modes, by introducing new annihilation operators

$$
\hat{A}_{ \pm}=\hat{A}_{X} \mp \mathrm{i} \hat{A}_{Y}
$$

The new modes are characterised by opposite generalised angular momenta.

Now we couple the new modes to electrons through a linear coupling $-\left(\hat{X} \hat{F}_{X}+\hat{Y} \hat{F}_{Y}\right)$, where $\hat{F}_{X}, \hat{F}_{Y}$ are electronic operators yielding the atomic forces on the two respective modes. Finally we compute the rates of change of the populations of the new modes, $N_{ \pm}$. The result is [8]

$$
\begin{aligned}
\dot{N}_{ \pm} & =\frac{\pi}{2 M \omega}\left(N_{ \pm}+1\right) \sum_{\alpha, \beta} f_{\alpha}\left(1-f_{\beta}\right)\left|\left\langle\psi_{\beta}\left|\left(\hat{F}_{X} \mp \mathrm{i} \hat{F}_{Y}\right)\right| \psi_{\alpha}\right\rangle\right|^{2} \delta\left(E_{\beta}-E_{\alpha}+\hbar \omega\right) \\
& -\frac{\pi}{2 M \omega} N_{ \pm} \sum_{\alpha, \beta} f_{\alpha}\left(1-f_{\beta}\right)\left|\left\langle\psi_{\beta}\left|\left(\hat{F}_{X} \pm \mathrm{i} \hat{F}_{Y}\right)\right| \psi_{\alpha}\right\rangle\right|^{2} \delta\left(E_{\beta}-E_{\alpha}-\hbar \omega\right)
\end{aligned}
$$

where $\left\{\left|\psi_{\alpha}\right\rangle\right\}$ are the eigenstates of a chosen phonon-free one-electron Hamiltonian, with eigenenergies $\left\{E_{\alpha}\right\}$ and populations $\left\{f_{\alpha}\right\}, \omega$ is the common frequency of the two degenerate vibrational modes, and $M$ is their effective mass.

From this expression, Ref. [8] then shows how above a critical bias, such as to overcome the electronic friction force, the current in a nanoscale conductor drives one of the two modes, with an exponential growth in energy and angular momentum, and damps the other (depending on the sign of the bias). This is the atomic waterwheel effect, which can further be related to the work 
done by non-conservative current-induced forces, described by a non-hermitian effective dynamical response matrix [6].

Here we pursue a different route. First we rearrange equation (2) as

$$
\begin{aligned}
\dot{N}_{ \pm} & =\frac{\pi}{2 M \omega} \sum_{\alpha, \beta} f_{\alpha}\left(1-f_{\beta}\right)\left|\left\langle\psi_{\beta}\left|\left(\hat{F}_{X} \mp \mathrm{i} \hat{F}_{Y}\right)\right| \psi_{\alpha}\right\rangle\right|^{2} \delta\left(E_{\beta}-E_{\alpha}+\hbar \omega\right) \\
& -\frac{\pi}{2 M \omega} N_{ \pm} \sum_{\alpha, \beta}\left(f_{\beta}-f_{\alpha}\right)\left|\left\langle\psi_{\beta}\left|\left(\hat{F}_{X} \mp \mathrm{i} \hat{F}_{Y}\right)\right| \psi_{\alpha}\right\rangle\right|^{2} \delta\left(E_{\beta}-E_{\alpha}+\hbar \omega\right) .
\end{aligned}
$$

The first term describes spontaneous phonon emission by electrons [8]. We are interested here in the second term, which describes stimulated processes.

Usually the overall prefactor of $N_{ \pm}$in the RHS of equation (3) is negative. Then the second term describes normal damping of the atomic motion, due to the electronic friction. This enables the phonons to equilibrate with the electrons - to a common temperature at equilibrium, or an elevated temperature under current, due to Joule heating. However, in the atomic waterwheel effect, this prefactor becomes positive for one of the two modes, resulting in uncompensated stimulated emission of directional phonons - an equivalent way of thinking of the action of the non-conservative current-induced forces $[6,8]$.

Our aim now is to consider whether - and under what conditions - this could happen in bulk conduction. We will consider the simplest case of a free-electron conductor (jellium). As our starting phonon modes $X$ and $Y$ we can always select two frequency-degenerate standing waves, with displacements proportional to $\cos (\mathbf{q} \cdot \mathbf{R})$ and $\sin (\mathbf{q} \cdot \mathbf{R})$, respectively, at position $\mathbf{R}$ [7]. Then the new modes \pm are waves travelling in opposite directions, with wave-vectors $\pm \mathbf{q}$. The states $\left\{\left|\psi_{\alpha}\right\rangle\right\}$ are plane waves $\{|\mathbf{k}\rangle\}$ with dispersion $E(\mathbf{k})=\hbar^{2} k^{2} / 2 m$. Selecting mode + for definiteness, the expression for the stimulated contribution to the rate of change of $N_{\mathbf{q}} \equiv N_{+}$takes the form

$$
\dot{N}_{\mathbf{q}}=-\frac{\pi}{2 M \omega} N_{\mathbf{q}} \sum_{\mathbf{k}, \mathbf{k}^{\prime}}\left[f\left(\mathbf{k}^{\prime}\right)-f(\mathbf{k})\right]\left|F\left(\mathbf{k}^{\prime}, \mathbf{k}\right)\right|^{2} \delta\left(E\left(\mathbf{k}^{\prime}\right)-E(\mathbf{k})+\hbar \omega\right) \delta\left(\mathbf{k}^{\prime}+\mathbf{q}-\mathbf{k}\right),
$$

where $F\left(\mathbf{k}^{\prime}, \mathbf{k}\right)$ is a scattering amplitude whose precise form we need not specify.

To describe steady-state current, we adopt the usual solution to the Boltzmann equation in the relaxation-time approximation describing a shifted Fermi sphere:

$$
f(\mathbf{k})=f_{0}\left(\mathbf{k}-\mathbf{k}_{0}\right),
$$

where $f_{0}(\mathbf{k})$ is the equilibrium Fermi-Dirac distribution and $\hbar \mathbf{k}_{0}=m \mathbf{v}_{0}$, with $\mathbf{v}_{0}$ being the drift velocity of the current-carrying electron gas.

By analogy with the atomic waterwheel effect, we examine the sign of the overall prefactor of $N_{\mathbf{q}}$ on the right in (4). Specifically, we examine the possibility $f(\mathbf{k})>f\left(\mathbf{k}^{\prime}\right) \Rightarrow f_{0}\left(\mathbf{k}-\mathbf{k}_{0}\right)>f_{0}\left(\mathbf{k}^{\prime}-\mathbf{k}_{0}\right)$, 
which would tend to make the prefactor positive, resulting in exponential growth of the phonon population. This requires $E\left(\mathbf{k}-\mathbf{k}_{0}\right)<E\left(\mathbf{k}^{\prime}-\mathbf{k}_{0}\right)$. Together with the energy- and momentumconservation conditions

$$
E(\mathbf{k})=E\left(\mathbf{k}^{\prime}\right)+\hbar \omega \quad, \quad \mathbf{k}=\mathbf{k}^{\prime}+\mathbf{q}
$$

this gives

$$
\omega<\mathbf{q} \cdot \mathbf{v}_{0}
$$

The threshold condition for uncompensated stimulated emission by the current therefore is

$$
v_{0}>c,
$$

where $c=\omega / q$ is the speed of sound in the jellium metal.

The current density corresponding to the critical drift velocity in (8), for typical physical parameters, is in the region of $10^{13} \mathrm{Am}^{-2}$. This is huge for a macroscopic metal. We will return to this question later. However for atomic wires this is a modest current density. We therefore set out to illustrate the resultant dynamics in long atomic chains.

\section{NON-CONSERVATIVE DYNAMICS IN ATOMIC WIRES}

To study non-conservative dynamics in long atomic wires, we adopt the quasi-classical description of current-driven atomic motion in Ref. [9]. It maps harmonic quantum phonons, weakly coupled to a non-equilibrium electron bath, onto an effective ensemble of classical trajectories generated by a Langevin-type equation of the form

$$
M_{i} \ddot{X}_{i}=-\sum_{j} K_{i j} X_{j}+\sum_{j} L_{i j} \dot{X}_{j}+f_{i}
$$

for vibrational degree of freedom $X_{i}$ of mass $M_{i}$. The effective stochastic force $f_{i}$ holds the quantum mechanics of the problem and describes - in general - coloured noise, with equilbrium and current-induced contributions. Omitting $f_{i}$ corresponds to suppressing spontaneous emission and is equivalent to Ehrenfest dynamics. We will do so here as it does not contribute to our main argument.

$K_{i j}$ is a non-hermitian effective non-equilibrium dynamical response matrix [6]. Its antihermitian part describes the non-conservative current-induced forces. $L_{i j}$ is also composed of a hermitian and an anti-hermitian part [9]. The hermitian part describes the electronic friction; the anti-hermitian part comes solely from current flow and is related to the Berry phase [10]. 
A limitation of equation (9) is that it employs a perturbative description of the electron-phonon interaction which - at the simplest level adopted here - does not allow the coefficients $K_{i j}$ and $L_{i j}$ to respond to the changes in current caused by the response of the atomic motion to the currentinduced forces. However, it is sufficient for a qualitative illustration of the phenomena we are interested in.

Here we retain the dominant forces, which come from the full $K_{i j}$ and the equilibrium part of the friction. To solve the model equations of motion we adopt a single-orbital tight-binding model fitted to the elastic properties of bulk gold. We consider a 200-atom long dynamical atomic chain, sandwiched between perfect $1 \mathrm{~d}$ electrodes. The geometry and parameters are the same as in Ref. [6]. The calculation of $K_{i j}$ is described in Ref. [6], and that of the friction matrix - in Ref. [9].

The atomic motion starts from the relaxed atomic positions at zero bias, with small random velocities. Figure 2 shows the modulus of the Fourier components of the atomic displacements in time, under a bias large enough to overcome the electronic friction forces. We clearly see the exponentially growing peaks corresponding to travelling phonons in the structure. Figure 3

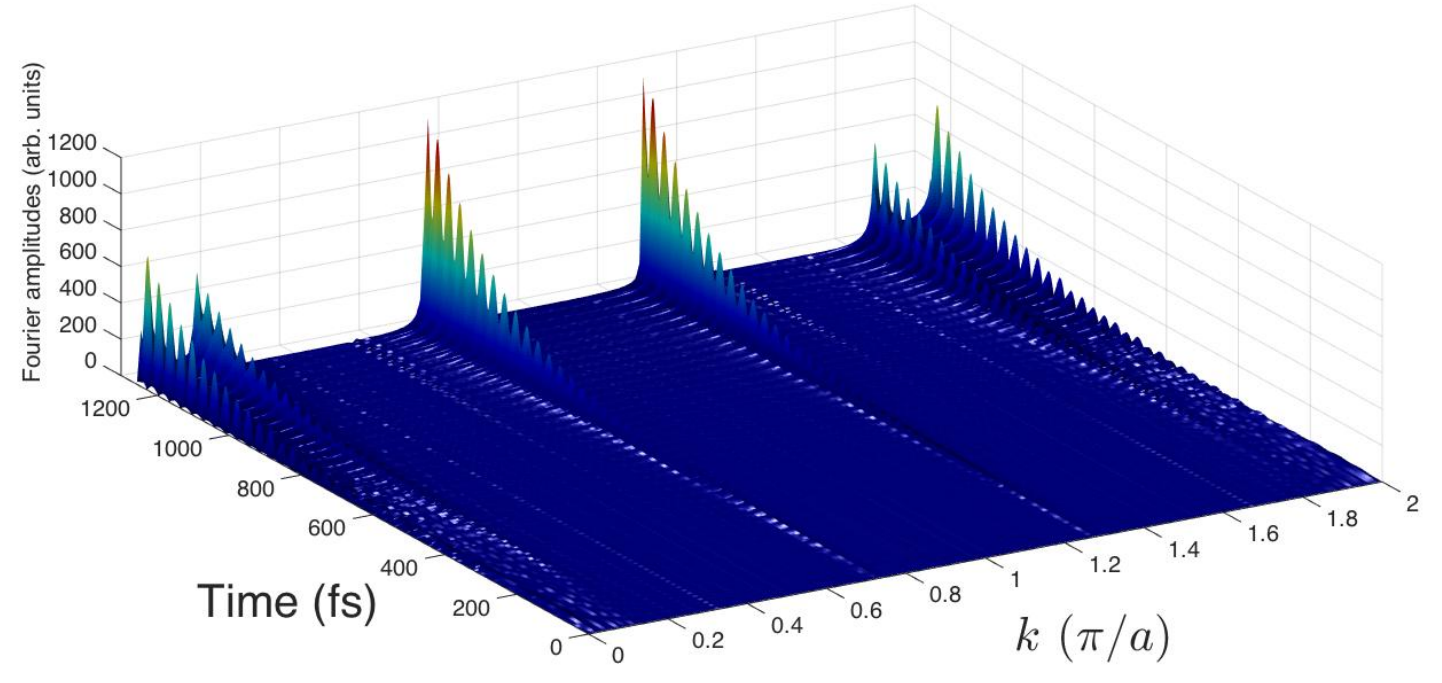

FIG. 2: Modulus of the Fourier spectrum of the longitudinal atomic displacements in a current-carrying atomic chain as a function of time. ( $a$ is the lattice parameter.)

compares the Fourier spectrum soon after the start of the simulation with that a few tens of vibration periods later.

Travelling and standing vibrations are distinguished by the fact that for standing phonons the Fourier components reach down to zero periodically in time. Indeed, the corrugation of the rising 


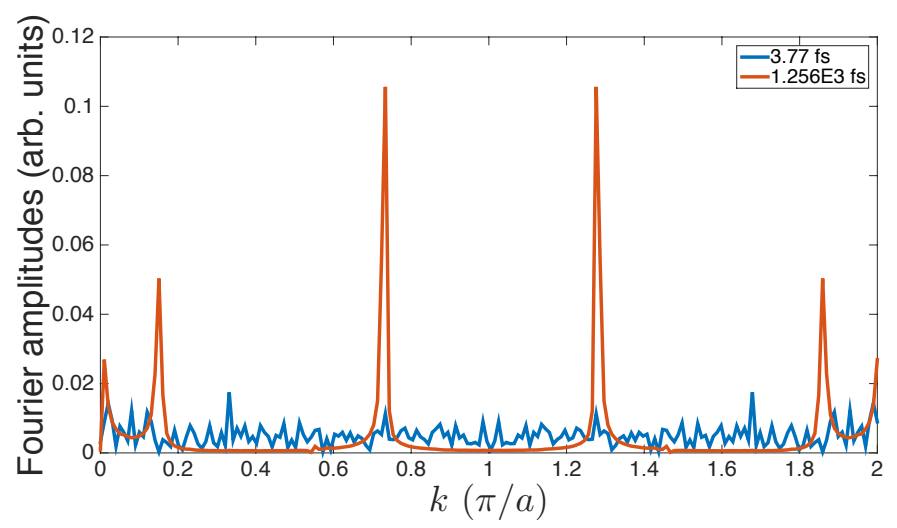

FIG. 3: Comparison of the Fourier spectra from Fig. 2 at two individual times.

crests on the Fourier spectrum indicates a significant standing-wave admixture in the motion. This can be seen more clearly in Fig. 4, which shows the data from Fig. 2 normalised by the area under the spectrum. The standing waves in the present case are an unavoidable consequence of the



FIG. 4: The data from Fig. 2, normalised by the area under the spectrum.

rigid-end boundary conditions, and result from internal reflections off the fixed ends of the wire.

The directionality of the travelling waves, resulting from the uncompensated stimulated emission under the non-conservative current-induced forces, can be seen from a real-space plot of the motion [6]. Fig. 5 displays the atomic motion over a window of $200 \mathrm{fs}$, some time into the simulation. The generated right-travelling waves are clearest near the left end of the wire, where the incoming 
particle current is entering the dynamical region.



FIG. 5: Atomic displacements (in $\AA$ ) versus atomic position and time, during the time-window from 600 fs to $800 \mathrm{fs}$.

Of course in reality the exponential growth cannot continue for ever. The limiting factor which eventually enables saturation of the atomic kinetic energy is the increase in resistance - and decrease in current - with increasing atomic amplitude. This requires the full non-adiabatic open-boundary molecular dynamics of Ref. [11], as opposed to the perturbative description of equation (9).

\section{CONCLUDING REMARKS}

We have shown that non-conservative current-induced forces can result in uncompensated stimulated phonon emission - with potentially dramatic effects - in extended conductors, and that this effect is associated with a threshold current density. However the threshold condition in (8) corresponds to current densities that are too high to be relevant to normal macroscopic conductors. Can the effect still occur, and could it be a contributing factor to effects such as electroplasticity $[12] ?$

We cannot give a conclusive answer to this question. Indeed, it is hoped that the present paper would stimulate further work on this problem. We conclude, however, by observing that the details of the Fermi surface may play an important role. Retaining a spherical Fermi surface, but allowing 
a general dispersion, $E=E(k)$, generalises (8) to, approximately,

$$
\frac{j}{e \hbar v_{F} k_{F} D_{F} / 3}>c
$$

where $j$ is the current density, and $v_{F}, k_{F}$ and $D_{F}$ are the Fermi velocity, wave-vector and density of states per unit volume, respectively. Thus variations in the Fermi properties of the electrons could influence significantly the critical bulk current density for the non-conservative effect.

\section{Acknowledgements}

We are grateful to Adrian Sutton for helpful discussions.

1 J. C. Cuevas and E. Scheer: 'Molecular Electronics: An Introduction to Theory and Experiment', World Scientific, 2010.

2 T. N. Todorov, J. hoekstra and A. P. Sutton: 'Current-induced forces in atomic-scale conductors', Phil. Mag. B, 2000, 80, 421-455.

3 T. N. Todorov: 'Local heating in ballistic atomic-scale contacts', Phil. Mag. B, 1998, 77, 965-973.

4 R. S. Sorbello: 'Theory of electromigration', Solid State Phys., 1997, 51, 159-231.

5 D. Dundas, E. J. McEniry and T. N. Todorov: 'Current-driven atomic waterwheels', Nat. Nanotechnology, 2009, 4, 99-102.

6 B. Cunningham, T. N. Todorov and D. Dundas: 'Nonconservative current-driven dynamics: beyond the nanoscale', Beilstein J. Nanotechnol., 2015, 6, 2140-2147.

7 J.-T. Lü, R. B. Christensen, J.-S. Wang, P. Hedegård and M. Brandbyge: 'Current-Induced Forces and Hot Spots in Biased Nanojunctions', Phys. Rev. Lett., 2015, 114, 096801.

8 T. N. Todorov, D. Dundas, A. T. Paxton and A. P. Horsfield: 'Nonconservative current-induced forces: A physical interpretation', Beilstein J. Nanotechnol., 2011, 2, 727-733.

9 J.-T. Lü, M. Brandbyge, P. Hedegård, T. N. Todorov and D. Dundas: 'Current-induced atomic dynamics, instabilities, and Raman signals: Quasiclassical Langevin equation approach', Phys. Rev. B, 2012, 85, 245444 .

10 J.-T. Lü, M. Brandbyge and P. Hedegård: 'Blowing the fuse: Berry's phase and runaway vibrations in molecular conductors', Nano Lett., 2010, 10, 1657-1663.

11 B. Cunningham, T. N. Todorov and D. Dundas: 'Nonconservative dynamics in long atomic wires', Phys. Rev. B, 2014, 90, 115430.

12 S. D. Antolovich and H. Conrad: 'The effects of Electric Currents and Field on Deformation in Metals, Ceramics, and Ionic Materials: An Interpretive Survey', Materials and Manufacturing Processes, 2004, 19, 587-610. 